\title{
Cerebellum Can Serve As a Pseudo-Reference Region in Alzheimer Disease to Detect Neuroinflammation Measured with PET Radioligand Binding to Translocator Protein
}

\author{
Chul Hyoung Lyoo ${ }^{1,2}$, Masamichi Ikawa ${ }^{2}$, Jeih-San Liow ${ }^{2}$, Sami S. Zoghbi², Cheryl L. Morse ${ }^{2}$, Victor W. Pike ${ }^{2}$, \\ Masahiro Fujita ${ }^{2}$, Robert B. Innis ${ }^{2}$, and William Charles Kreisl ${ }^{2,3}$ \\ ${ }^{1}$ Department of Neurology, Gangnam Severance Hospital, Yonsei University College of Medicine, Seoul, South Korea; ${ }^{2}$ Molecular \\ Imaging Branch, National Institute of Mental Health, National Institutes of Health, Bethesda, Maryland; and ${ }^{3}$ Taub Institute for \\ Research on Alzheimer's Disease and the Aging Brain, Columbia University Medical Center, New York, New York
}

Alzheimer disease (AD) is associated with an increase in the brain of the 18-kDa translocator protein (TSPO), which is overexpressed in activated microglia and reactive astrocytes. Measuring the density of TSPO with PET typically requires absolute quantitation with arterial blood sampling, because a reference region devoid of TSPO does not exist in the brain. We sought to determine whether a simple ratio method could substitute for absolute quantitation of binding with ${ }^{11} \mathrm{C}-\mathrm{PBR} 28$, a second-generation radioligand for TSPO. Methods: ${ }^{11} \mathrm{C}$-PBR28 PET imaging was performed in 21 healthy controls, 11 individuals with mild cognitive impairment, and $25 \mathrm{AD}$ patients. Group differences in ${ }^{11} \mathrm{C}$-PBR28 binding were compared using 2 methods. The first was the gold standard method of calculating total distribution volume $\left(V_{\mathrm{T}}\right)$, using the 2-tissue-compartment model with the arterial input function, corrected for plasma-free fraction of radiotracer $\left(f_{\mathrm{P}}\right)$. The second method used a ratio of brain uptake in target regions to that in cerebellum - that is, standardized uptake value ratio (SUVR). Results: Using absolute quantitation, we confirmed that TSPO binding $\left(V_{\mathrm{T}} / f_{\mathrm{P}}\right)$ was greater in $\mathrm{AD}$ patients than in healthy controls in expected temporoparietal regions and was not significantly different among the 3 groups in the cerebellum. When the cerebellum was used as a pseudo-reference region, the SUVR method detected greater binding in $A D$ patients than controls in the same regions as absolute quantification and in 1 additional region, suggesting SUVR may have greater sensitivity. Coefficients of variation of SUVR measurements were about two-thirds lower than those of absolute quantification, and the resulting statistical significance was much higher for SUVR when comparing $A D$ and healthy controls (e.g., $P<0.0005$ for SUVR vs. $P=0.023$ for $V_{\mathrm{T}} / f_{\mathrm{P}}$ in combined middle and inferior temporal cortex). Conclusion: To measure TSPO density in AD patients and control subjects, a simple ratio method SUVR can substitute for, and may even be more sensitive than, absolute quantitation. The SUVR method is expected to improve subject tolerability by allowing shorter scanning time and not requiring arterial catheterization. In addition, this ratio method allows smaller sample sizes for comparable statistical significance because of the relatively low variability of the ratio values.

\footnotetext{
Received Oct. 23, 2014; revision accepted Feb. 3, 2015.

For correspondence or reprints contact: William Charles Kreisl, Taub Institute, Department of Neurology, 622 W. 168th St., PH 19th Floor, New York, NY 10032

E-mail: kreislw@mail.nih.gov

Published online Mar. 12, 2015.

COPYRIGHT (C 2015 by the Society of Nuclear Medicine and Molecular Imaging, Inc.
}

Key Words: Alzheimer disease; neuroinflammation; ${ }^{11} \mathrm{C}-\mathrm{PBR} 28$; positron emission tomography; ratio method

J Nucl Med 2015; 56:701-706

DOI: 10.2967/jnumed.114.146027

\section{A \\ lzheimer disease $(\mathrm{AD})$ is associated with neuroinflamma-} tion characterized by activated microglia and reactive astrocytes. Because these reactive neuroimmune cells overexpress the $18-\mathrm{kDa}$ translocator protein (TSPO), TSPO density has been used as a biomarker for neuroinflammation in $\mathrm{AD}$ and other neurologic diseases (1). We recently found that TSPO binding was greater in $\mathrm{AD}$ patients than in age-matched controls or patients with mild cognitive impairment (MCI) who had a positive amyloid scan result (2). That is, increased TSPO binding appeared to mark, and might play a pathophysiologic role in, the transition from MCI to AD.

A major barrier to expanding studies that use TSPO imaging to assess the potential role of inflammation in $\mathrm{AD}$ is that PET measurements of TSPO density typically require absolute quantitation relative to the concentration of radioligand in arterial blood. This measurement of plasma concentrations adds error to the final values, requires significant equipment and expertise, and entails arterial catheterization of the subject. A relative method of measurement (e.g., one brain region compared with another) would be expected to have smaller variability than an absolute value, in part because of cancellation of global scale changes or errors in measurement of plasma concentrations. However, relative measurement requires either a reference or a pseudo-reference region. Unfortunately, a true reference region (i.e., devoid of TSPO) does not exist in the brain, because TSPO is present in gray matter, white matter, vessel walls, and even choroid plexus $(3,4)$. A pseudo-reference region would contain TSPO but not differ between comparison groups. Such a region could, for example, be used to compare the ratio of target to pseudo-reference region in patients versus controls. However, this approach requires that prior studies using absolute quantitation confirmed that the pseudo-reference region does not significantly differ between patients and controls. To date, no study has identified or proved that a pseudo-reference region exists for second-generation TSPO imaging in AD, including white matter. 
Using absolute quantitation, we previously found that TSPO binding in the cerebellum, measured with ${ }^{11} \mathrm{C}-\mathrm{PBR} 28$, did not differ between $\mathrm{AD}$ patients and either healthy controls (HCs) or MCI subjects (2). This finding is consistent with the cerebellum of $\mathrm{AD}$ patients being relatively spared of pathology, including inflammation (5-7), and suggests that the cerebellum could be used as a pseudo-reference region as an alternative to absolute quantification.

This study sought to determine whether the method of absolute quantitation that requires arterial blood sampling could be substituted with a simple method that uses only the ratio of brain radioactivity in a target region compared with that in the cerebellum. We recruited 15 more subjects than in our previous report (2) and then measured TSPO binding in 25 AD patients, $21 \mathrm{HCs}$, and 11 patients with MCI. We compared 2 methods of analysis, absolute quantitation of receptor binding, which requires an arterial input function, and relative quantitation of receptor binding, which requires only PET images and was calculated as the ratio of brain uptake in target regions compared with that in the cerebellum (i.e., standardized uptake value ratio [SUVR]).

\section{MATERIALS AND METHODS}

\section{Subjects}

Subjects included $21 \mathrm{HCs}$ (15 men and 6 women; mean age \pm $\mathrm{SD}, 55.1 \pm 15.3 \mathrm{y}), 11 \mathrm{MCI}$ patients $(7$ men and 4 women; mean age $\pm \mathrm{SD}, 72.2 \pm 9.3 \mathrm{y}$ ), and $25 \mathrm{AD}$ patients $(11 \mathrm{men}$ and 14 women; mean age $\pm \mathrm{SD}, 63.0 \pm 8.3 \mathrm{y}$ ). All patients were amyloid-positive on PET imaging with ${ }^{11} \mathrm{C}$-Pittsburgh compound $\mathrm{B}$ $\left({ }^{11} \mathrm{C}\right.$-PIB) based on criteria used in our earlier study (2). AD patients met updated criteria for probable AD dementia with evidence of AD pathophysiologic process (8), and MCI patients met updated criteria for MCI due to high or intermediate likelihood of developing AD (9). Some subjects were included in our previously published study (2). Binding affinity status (high, mixed, or low) was determined using leukocyte binding assay as previously described $(2,10)$, and low-affinity binders were excluded from the study. There were 7 high-affinity binders (HABs) and 14 middleaffinity binders (MABs) among $\mathrm{HCs}, 5 \mathrm{HABs}$ and 6 MABs among MCI patients, and $11 \mathrm{HABs}$ and $14 \mathrm{MABs}$ among AD patients. Both radioligand preparation and acquisition and processing of ${ }^{11}$ C-PBR28 PET and MR images are described in the supplemental materials (available online at http://jnm.snmjournals.org). PET images were not corrected for partial-volume effects (PVEs).

This study was approved by the Combined Neuroscience Institutional Review Board of the National Institutes of Health Intramural Research Program. All subjects or their surrogate provided written informed consent to participate.

\section{Estimation of Binding Values}

All kinetic analyses were performed with the PKIN module in PMOD 3.1 (PMOD Technologies Ltd.). The consensus nomenclature of reversible binding radioligands was followed (11).

Metabolite-corrected plasma input function and whole-blood radioactivity were fitted to a triexponential function. The time delay from the radial artery to the brain was calculated with the whole-blood radioactivity curve and whole-gray matter time-activity curve. A model curve was fitted to regional time-activity curves with a metabolite-corrected input function using the 2-tissue-compartment model, as described previously (12). Finally, total distribution volume $\left(V_{\mathrm{T}}\right)$ was calculated using the rate constants $\left(K_{1}, k_{2}, k_{3}\right.$, and $\left.k_{4}\right)$ estimated from this model and corrected for plasma-free fraction of radioligand $\left(f_{\mathrm{P}}\right)$.
For a noninvasive measure of ${ }^{11} \mathrm{C}-\mathrm{PBR} 28$ binding, we created time-averaged images from 30-min intervals of scan data (10-40, $20-50,30-60,40-70,50-80$, and $60-90 \mathrm{~min}$ ) and converted the measured activity to standardized uptake value (SUV) by multiplying subject body weight and dividing by injected activity. Regional SUVs were measured by overlaying the volume-of-interest mask using the same regions of interest studied in our prior report (2), and regional SUVRs were calculated by dividing the SUV of each region by cerebellar SUV. We sought to determine which time points best provided SUVRs, similar to the determination made for ${ }^{11} \mathrm{C}$-PIB by Price et al. (13). Unlike ${ }^{11} \mathrm{C}$-PIB, which shows a stable ratio of radioactivity in the cerebellum to plasma in late time points of the PET scan (13), for ${ }^{11} \mathrm{C}-\mathrm{PBR} 28$ this ratio increased until the end of the scan without plateauing. Therefore, to acquire an optimal time interval for SUVR, we performed a linear regression analysis between $V_{\mathrm{T}} / f_{\mathrm{P}}$ values calculated from the entire 90 -min scan and SUVRs calculated from sequential 30-min time intervals of the image data from the combined middle and inferior temporal cortex (Supplemental Fig. 1). Because the best correlation was achieved with data from 60 to $90 \mathrm{~min}(r=0.35, P=0.008)$, we used $60-$ $90 \mathrm{~min}$ as the time interval for obtaining SUVRs for the remainder of the analysis. We also compared group binding using distribution volume ratio (DVR) by dividing the $V_{\mathrm{T}}$ of each target region by $V_{\mathrm{T}}$ of the cerebellar pseudo-reference region.

\section{Statistical Analysis}

SPSS 11.5 (SPSS Inc.) was used for the statistical analysis. Statistical correction for TSPO genotype was performed according to previously published methods (2). For $V_{\mathrm{T}} / f_{\mathrm{P}}$, SUVR, and DVR, TSPO genotype was used as a fixed factor to correct for affinity differences caused by the rs6971 single nucleotide polymorphism (SNP). For SUVR, statistical analysis was also performed without $T S P O$ genotype correction. Benjamini-Hochberg's false-discovery rate with a threshold $P$ value of 0.05 was used to correct for regionwise multiple comparisons (14). Bonferroni adjustment was used for multiple comparisons of each between-group comparison. The variability of binding values was defined as coefficient of variation $(\% \mathrm{COV})=\mathrm{SD} /$ mean $\times 100 \%$. For correlative analyses, correlations were first run between the individual outcome measures and TSPO genotype. Standardized residuals were then plotted against each other. To determine whether both $V_{\mathrm{T}} / f_{\mathrm{P}}$ and SUVRs correlate with clinical severity of AD, we performed correlative analysis with the Clinical Dementia Rating scale sum-of-boxes scores as independent variables and ${ }^{11} \mathrm{C}-\mathrm{PBR} 28$ binding values in combined middle and inferior temporal cortex as dependent variables.

\section{RESULTS}

We used the cerebellar gray matter segmented by FreeSurfer (http://surfer.nmr.mgh.harvard.edu) to measure uptake in the pseudoreference tissue. The mean volume of the cerebellar gray matter of $\mathrm{HCs}$ was slightly greater than that of MCI and $\mathrm{AD}$ patients $(\mathrm{HCs}$, $96.5 \pm 12.0 \mathrm{~cm}^{3}$; MCI, $95.8 \pm 9.4 \mathrm{~cm}^{3}$; and $\mathrm{AD}, 91.3 \pm 9.8 \mathrm{~cm}^{3}$ ). However, no statistically significant difference was observed in cerebellar gray matter volume among the 3 groups, either with or without correction for age.

No differences in $V_{\mathrm{T}}$ values were observed between the groups (Supplemental Table 1). In contrast, and consistent with results from our earlier study (2), AD patients showed greater $V_{\mathrm{T}} / f_{\mathrm{P}}$ values than controls in the inferior parietal, combined middle and inferior temporal, and entorhinal cortices $(P<0.05$; Table 1 ; Fig. 1; Supplemental Table 1). AD patients showed greater $V_{\mathrm{T}} / f_{\mathrm{P}}$ 
TABLE 1

Effect of Quantification Method on Level of Statistical Significance in Detecting Differences in ${ }^{11} \mathrm{C}-\mathrm{PBR} 28$ Binding

\begin{tabular}{|c|c|c|c|}
\hline \multirow[b]{2}{*}{ Region } & \multicolumn{3}{|c|}{$P(\mathrm{AD}$ vs. $\mathrm{HC})$} \\
\hline & $V_{\mathrm{T}} / f_{\mathrm{P}}$ & SUVR & DVR \\
\hline Inferior parietal & 0.028 & $<0.0005$ & $<0.00005$ \\
\hline $\begin{array}{l}\text { Middle and inferior } \\
\text { temporal }\end{array}$ & 0.023 & $<0.0005$ & $<0.00005$ \\
\hline Precuneus & NS & $0.048^{*}$ & 0.006 \\
\hline Entorhinal & 0.048 & 0.009 & 0.001 \\
\hline Parahippocampal & NS & 0.006 & 0.009 \\
\hline
\end{tabular}

${ }^{*}$ Region did not survive regionwise correction for multiple comparisons.

NS $=$ not significant

$P$ values were derived from univariate ANOVA using diagnosis and TSPO genotype as fixed factors.

values than MCI patients in the entorhinal and combined middle and inferior temporal cortices. All these regions survived correction for multiple comparisons. Cerebellar $V_{\mathrm{T}} / f_{\mathrm{P}}$ and SUVs did not differ among controls, MCI patients, and AD patients $(P>0.05$; Table 1; Fig. 2).

SUVRs for ${ }^{11} \mathrm{C}-\mathrm{PBR} 28$ were greater in $\mathrm{AD}$ patients than controls in the inferior parietal and combined middle and inferior temporal cortices; precuneus; and entorhinal and parahippocampal cortices (Table 1; Fig. 1). With the exception of the precuneus, these regions survived correction for multiple comparisons. The SUVRs of the combined middle and inferior temporal cortex were greater in $\mathrm{AD}$ patients than MCI patients and survived correction for multiple comparisons. Both $V_{\mathrm{T}} / f_{\mathrm{P}}$ values and SUVRs in the combined middle and inferior temporal cortex were positively correlated with the Clinical Dementia Rating sum-of-boxes scores $(P<0.01$; Fig. 3).

The variability of SUVRs (\%COV) was much lower than that of $V_{\mathrm{T}} / f_{\mathrm{P}}$ values in every region and every diagnostic group (Table 2 ). Also, within the same TSPO genotype groups, the variability of SUVRs was much lower than that of $V_{\mathrm{T}} / f_{\mathrm{P}}$ values $(\% \mathrm{COV}$ of SUVR for $\mathrm{HAB}, 1 \%-9 \%$, and $\mathrm{MAB}, 4 \%-13 \%$, vs. $\% \mathrm{COV}$ of $V_{\mathrm{T}} / f_{\mathrm{P}}$ for $\mathrm{HAB}, 13 \%-27 \%$, and $\mathrm{MAB}, 16 \%-36 \%$ ).
DVR values were greater in patients with $\mathrm{AD}$ than controls in the inferior parietal lobule, combined middle and inferior temporal cortex, precuneus, hippocampus, entorhinal cortex, and parahippocampal gyrus $(P<0.01)$. DVR values were greater in $\mathrm{AD}$ patients than MCI patients in the inferior parietal lobule, middle and inferior temporal cortex, occipital cortex, entorhinal cortex, and parahippocampal gyrus $(P<0.04)$ (Supplemental Table 1). Variability was similar for both SUVRs and DVR values after genotype correction (\%COV, $7 \%-21 \%$ vs. $8 \%-$ $25 \%)$.

SUVRs for ${ }^{11} \mathrm{C}-\mathrm{PBR} 28$ were greater in AD patients than controls even without genotype correction in the inferior parietal $(P=0.002)$ and combined middle and inferior temporal cortices $(P=0.003)$. However, the statistical significance was greater $(P<0.0005$ for 2 regions above), and 2 more regions (entorhinal and parahippocampal cortices) became significant, after correcting for TSPO genotype. Paradoxically, mean SUVRs in HABs were consistently lower than those in MABs in all diagnostic groups and in all regions (mean SUVRs of combined middle and inferior temporal cortex [HAB vs. MAB]: 0.98 vs. 1.05 in HC, 0.98 vs. 1.10 in MCI, and 1.08 vs. 1.15 in AD; Supplemental Table 2; Supplemental Fig. 2).

\section{DISCUSSION}

This study found that a simple ratio method (SUVR) can substitute for, and may even be more sensitive than, absolute quantitation for ${ }^{11} \mathrm{C}-\mathrm{PBR} 28 \mathrm{PET}$. Therefore, this ratio method may be promising for the clinical application of ${ }^{11} \mathrm{C}-\mathrm{PBR} 28 \mathrm{PET}$ to study $\mathrm{AD}$. One may expect the ratio method to provide results independent of TSPO genotype because affinity status is the same in both the target regions and the cerebellum. However, because genotype differences affect specific binding and not nonspecific binding, this ratio method should not completely remove the effect of TSPO genotype on total binding values. Therefore, TSPO genotype correction is still recommended.

The major limitation shared by all tested second-generation TSPO radioligands is differential affinity for the target protein caused by the rs6971 (Ala147Thr) SNP in exon 4 of the TSPO gene (15). Individuals without the SNP demonstrate the expected affinity of radioligand for TSPO (high-affinity binding). In the case of ${ }^{11} \mathrm{C}-\mathrm{PBR} 28$, the presence of 1 copy of this SNP reduces specific TSPO binding by more than $40 \%$ (mixed-affinity binding); those homozygous for the SNP show no discernible binding at all (low-affinity binding) (10). Therefore, the gold standard method of quantification for second-generation TSPO radioligandscalculating the $V_{\mathrm{T}}$ using arterial input function (12)-requires correction for affinity status to avoid underestimating TSPO density in SNP carriers. Affinity status must be determined by genetic analysis or in vivo binding assay $(10,15)$.

However, even within the same affinity group, significant overlap in binding values exists $(10,16)$, suggesting that factors other than genotype contribute to this variability $(10,17,18)$. Altered TSPO expression in response to external stimuli (for instance, stress) as well as normal physiologic changes may partly explain
FIGURE 1. In combined middle and inferior temporal cortex, $V_{\mathrm{T}} / f_{\mathrm{P}}$, SUVR, and DVR values were greater for $\mathrm{AD}$ patients than for $\mathrm{MCl}$ patients or controls. Error bars denote mean $\pm \mathrm{SD}$. $\mathrm{SD}$ bars have similar heights for $V_{\mathrm{T}} / f_{\mathrm{P}}$ and SUVR due to different scales on the $y$-axes. Coefficient of variation of $V_{\mathrm{T}} / f_{\mathrm{P}}$ was 3-4 times greater than that for SUVR and DVR, as shown by \%COV values above SD bars. NS = not significant. Binding values are adjusted for TSPO genotype. 


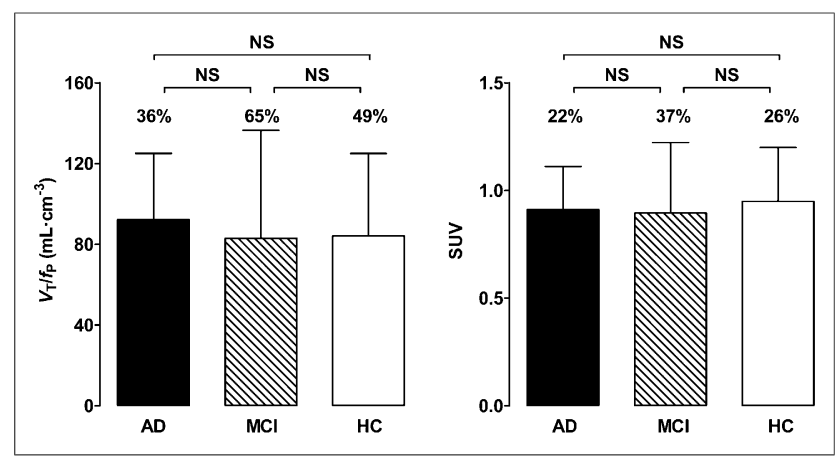

FIGURE 2. In cerebellum, ${ }^{11} \mathrm{C}-\mathrm{PBR} 28$ binding did not differ between controls and patients with $\mathrm{MCl}$ or $\mathrm{AD} .{ }^{11} \mathrm{C}$-PBR28 binding values in $V_{\mathrm{T}} / f_{\mathrm{P}}$ and SUV are shown. Error bars denote mean $\pm \mathrm{SD}$. \%COV values are shown above vertical bars. NS = not significant. Binding values are adjusted for TSPO genotype.

this individual variability $(19,20)$; it should also be noted that, even in the same subject, the contribution of these factors may change between scans. These factors may explain the low testretest reproducibility of the TSPO radioligands ${ }^{11} \mathrm{C}-(R)$ PK11195 and ${ }^{11}$ C-DPA713 using traditional analytic methods $(20,21)$. In addition, the 2-tissue-compartment model requires arterial catheterization to measure the concentration of parent radioligand in plasma. In addition to being invasive, arterial sampling adds a potential source of error that may increase PET data variability. The $f_{\mathrm{P}}$ may also contribute to variability in clinical PET studies. Therefore, a target-to-reference ratio approach may be preferable to absolute quantification with compartmental modeling, both for eliminating individual variation of physiologic TSPO expression and for avoiding errors in measuring plasma concentration of the radioligand. Our ratio approach appears to at least partly reduce the variability caused by differences in TSPO genotype as well. In the present study, the $\% \mathrm{COV}$ of $V_{\mathrm{T}} / f_{\mathrm{P}}$ was higher than $30 \%$ in all regions. This high variability requires relatively large sample sizes to detect statistically significant differences between groups. However, the variability of the SUVR was much lower $(<22 \%)$, and we expect this ratio method to increase statistical power to detect differences in TSPO binding in clinical studies of AD.

Errors in measuring the free fraction of radioligand may also contribute to high variability in clinical PET studies. Because only free radioligand enters the brain, correcting $V_{\mathrm{T}}$ for $f_{\mathrm{P}}$ should

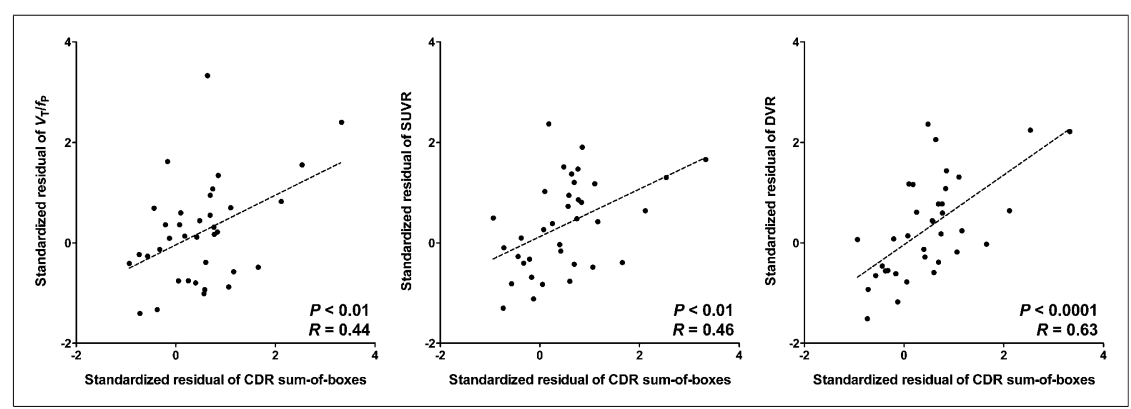

FIGURE 3. Correlation between binding values and severity of cognitive impairment measured by sum-of-boxes of Clinical Dementia Rating (CDR) score. $V_{T} / f_{\mathrm{P}}$, SUVR, and DVR of combined middle and inferior temporal cortex similarly correlated with severity of cognitive impairment. Noninvasive ratio method did not deteriorate correlation with clinical severity. theoretically increase the accuracy of PET measurement of receptor density. However, correcting for $f_{\mathrm{P}}$ may introduce noise, thereby reducing precision. In our study, we found no difference in the $\% \mathrm{COV}$ between $V_{\mathrm{T}}$ and $V_{\mathrm{T}} / f_{\mathrm{P}}$ values after genotype correction $(36 \%-74 \%$ vs. $34 \%-71 \%)$. Using $V_{\mathrm{T}} / f_{\mathrm{P}}$ thus did not reduce precision, suggesting that the theoretically more accurate $V_{\mathrm{T}} / f_{\mathrm{P}}$ should be used for absolute measurement of ${ }^{11} \mathrm{C}-\mathrm{PBR} 28$ binding.

For a ratio method to be clinically useful in PET studies, the reference region should be relatively unaffected by disease pathology in terms of the density of the target protein. Although diffuse amyloid plaques with surrounding microglia can be found in the cerebellum in advanced AD, the morphology of the cerebellar microglia differs from that of typical activated microglia in the neocortex, and the cerebellum is relatively spared from neurodegeneration (5-7). Therefore, we can reasonably assume that pathologic increases in TSPO are much lower in the cerebellum than in cortical gray matter regions most affected by $\mathrm{AD}$ and that the cerebellum can serve as the best reference region in $\mathrm{AD}$ (22). This argument is supported by the similar values observed for $V_{\mathrm{T}} / f_{\mathrm{P}}$ and SUV in the cerebellum among the HCs and MCI and AD patients in this and in our previous study (2).

When using ${ }^{11} \mathrm{C}-\mathrm{PBR} 28$, both DVR and SUVR discriminate AD patients from MCI patients and controls. Although the DVR method has the advantage of using the full kinetics of the radioligand, SUVR is more practical because it does not require arterial catheterization and allows for shorter scanning time.

An earlier study using the second-generation TSPO radioligand ${ }^{11} \mathrm{C}$-DAA1106 found greater binding in AD patients than controls in several regions, including the cerebellum (23). In that study, the cerebellar binding potential was $14 \%$ greater in $\mathrm{AD}$ patients than in controls (10 AD patients vs. 10 controls). In our larger study (25 AD patients vs. 21 controls), we found no difference in binding (using $V_{\mathrm{T}} / f_{\mathrm{P}}$ ) in the cerebellum. This discrepancy could be due to several factors, including the outcome measure used and the number and characteristics of the study subjects. However, the difference in the TSPO radioligand used could also be a factor. Therefore, studies using TSPO radioligands other than ${ }^{11} \mathrm{C}-\mathrm{PBR} 28$ should compare cerebellar binding using absolute values (e.g., $V_{\mathrm{T}} / f_{\mathrm{P}}$ ) between patient and control groups before using this SUVR method.

Our approach in this study was similar to that of Coughlin et al., who showed that normalizing the $V_{\mathrm{T}}$ values of regions for ${ }^{11} \mathrm{C}$ DPA713 to that of total gray matter lowered variability and improved test-retest reproducibility (20). This normalization also removed the effect of TSPO genotype differences. However, using global gray matter as the reference region means that binding in the target region is represented in both the numerator and the denominator. Therefore, this approach may underestimate binding in target regions, particularly relatively preserved areas in $\mathrm{AD}$, and may produce unexpected results of decreased binding in those regions in $\mathrm{AD}$ patients.

Unlike the gray matter normalization by Coughlin et al., SUVR with cerebellar pseudo-reference region did not completely ameliorate the TSPO genotype effect as 
TABLE 2

Effect of Quantification Method on Variability of ${ }^{11} \mathrm{C}-\mathrm{PBR} 28$ Binding Values

\begin{tabular}{|c|c|c|c|c|c|c|c|c|c|}
\hline \multirow[b]{3}{*}{ Region } & \multicolumn{9}{|c|}{$\% \mathrm{COV}$} \\
\hline & \multicolumn{3}{|c|}{$A D$} & \multicolumn{3}{|c|}{$\mathrm{MCl}$} & \multicolumn{3}{|c|}{$\mathrm{HC}$} \\
\hline & $V_{\mathrm{T}} / f_{\mathrm{P}}$ & SUVR & DVR & $V_{\mathrm{T}} / f_{\mathrm{P}}$ & SUVR & DVR & $V_{\mathrm{T}} / f_{\mathrm{P}}$ & SUVR & DVR \\
\hline Inferior parietal & 34.5 & 10.4 & 10.8 & 70.0 & 18.0 & 19.3 & 53.4 & 14.2 & 15.2 \\
\hline Middle and inferior temporal & 34.3 & 9.5 & 11.0 & 71.4 & 16.7 & 20.3 & 53.5 & 12.9 & 15.5 \\
\hline
\end{tabular}

larger differences between $\mathrm{AD}$ patients and controls were detected after correcting ${ }^{11} \mathrm{C}-\mathrm{PBR} 28$ binding values for affinity status. Therefore, we recommend genotype correction when using the SUVR method. In this study, TSPO genotype had a paradoxical effect on the ratio method of analysis, resulting in MABs having larger SUVRs than HABs. We are not certain if this unexpected finding represents a true result or not. Additional studies with larger sample sizes are needed to clarify the significance of this finding.

One of the most notable results of this study is clear replication of our previous findings. In our earlier study (2), we found that the AD patients showed greater $V_{\mathrm{T}} / f_{\mathrm{P}}$ values in the inferior parietal, entorhinal, and combined middle and inferior temporal cortices than controls when using PET data not corrected for PVE. By including 15 additional subjects (42 subjects in the previous vs. 57 in the current study), we found greater ${ }^{11} \mathrm{C}$-PBR2 8 binding in $\mathrm{AD}$ patients in the same regions. Moreover, using the SUVR method we found that $\mathrm{AD}$ patients showed greater binding than controls in a larger number of regions, with distribution similar to that seen in our previously reported results using ${ }^{11} \mathrm{C}-\mathrm{PBR} 28$ with a 2-tissue-compartment model and PVE-corrected data. Using SUVRs did not reduce the correlation between clinical severity and ${ }^{11} \mathrm{C}-\mathrm{PBR} 28$ binding seen with $V_{\mathrm{T}} / f_{\mathrm{P}}$. Although we did not include PVE correction in this study, we would expect to see even larger differences between AD patients and controls.

Because MCI patients with amyloid-positivity on PET are at high risk for developing dementia due to $\mathrm{AD}(24)$, the increased neuroinflammation observed with ${ }^{11} \mathrm{C}-\mathrm{PBR} 28$ PET may be a marker for conversion to clinical AD (2). We hope this SUVR method for ${ }^{11} \mathrm{C}-\mathrm{PBR} 28$ PET could prove useful for testing antiinflammatory agents in $\mathrm{AD}(25)$.

\section{CONCLUSION}

A simple ratio method (SUVR) can substitute for, and may even be more sensitive than, absolute quantitation for detecting regions with increased binding to TSPO in AD. This method reduces variability and has the advantages of not requiring arterial sampling and allowing for shorter scanning time. TSPO genotype correction was still required to increase sensitivity. This method is expected to improve subject tolerability for ${ }^{11} \mathrm{C}-\mathrm{PBR} 28$ PET studies in AD, particularly longitudinal studies, and increase the power necessary to detect group differences. The method also needs to be replicated in larger samples of AD patients before it can be widely used. For other diseases, application of this method will first require validation with gold standard methods of quantification that binding in the cerebellum is not affected by the disease itself.

\section{DISCLOSURE}

The costs of publication of this article were defrayed in part by the payment of page charges. Therefore, and solely to indicate this fact, this article is hereby marked "advertisement" in accordance with 18 USC section 1734 . This project was funded in part by the Intramural Research Program of the National Institute of Mental Health, National Institutes of Health (IRP-NIMH-NIH), and a fellowship award from the American Academy of Neurology Foundation, under clinicaltrials.gov identifier NCT00613119 (protocol \# 08-M-0066). No other potential conflict of interest relevant to this article was reported.

\section{ACKNOWLEDGMENT}

Ioline Henter provided excellent editorial assistance.

\section{REFERENCES}

1. Chen MK, Guilarte TR. Translocator protein $18 \mathrm{kDa}$ (TSPO): molecular sensor of brain injury and repair. Pharmacol Ther. 2008;118:1-17.

2. Kreisl WC, Lyoo $\mathrm{CH}, \mathrm{McGwier} \mathrm{M}$, et al. In vivo radioligand binding to translocator protein correlates with severity of Alzheimer's disease. Brain. 2013; 136:2228-2238.

3. Owen DR, Guo Q, Kalk NJ, et al. Determination of $\left[{ }^{11} \mathrm{C}\right] \mathrm{PBR} 28$ binding potential in vivo: a first human TSPO blocking study. J Cereb Blood Flow Metab. 2014;34:989-994.

4. Turkheimer FE, Edison $\mathrm{P}$, Pavese $\mathrm{N}$, et al. Reference and target region modeling of $\left[{ }^{11} \mathrm{C}\right]-(R)-\mathrm{PK} 11195$ brain studies. J Nucl Med. 2007;48:158167.

5. Braak H, Braak E. Neuropathological stageing of Alzheimer-related changes. Acta Neuropathol. 1991;82:239-259.

6. Mattiace LA, Davies P, Yen SH, Dickson DW. Microglia in cerebellar plaques in Alzheimer's disease. Acta Neuropathol. 1990;80:493-498.

7. Wood PL. The Cerebellum in AD. In: Wood PL, ed. Neuroinflammation. Totowa, New Jersey: Humana Press; 2003:295-300.

8. McKhann GM, Knopman DS, Chertkow H, et al. The diagnosis of dementia due to Alzheimer's disease: recommendations from the National Institute on AgingAlzheimer's Association workgroups on diagnostic guidelines for Alzheimer's disease. Alzheimers Dement. 2011;7:263-269.

9. Albert MS, DeKosky ST, Dickson D, et al. The diagnosis of mild cognitive impairment due to Alzheimer's disease: recommendations from the National Institute on Aging-Alzheimer's Association workgroups on diagnostic guidelines for Alzheimer's disease. Alzheimers Dement. 2011;7:270-279.

10. Kreisl WC, Jenko KJ, Hines CS, et al. A genetic polymorphism for translocator protein $18 \mathrm{kDa}$ affects both in vitro and in vivo radioligand binding in human brain to this putative biomarker of neuroinflammation. J Cereb Blood Flow Metab. 2013;33:53-58. 
11. Innis RB, Cunningham VJ, Delforge J, et al. Consensus nomenclature for in vivo imaging of reversibly binding radioligands. J Cereb Blood Flow Metab. 2007;27:1533-1539.

12. Fujita M, Imaizumi M, Zoghbi SS, et al. Kinetic analysis in healthy humans of a novel positron emission tomography radioligand to image the peripheral benzodiazepine receptor, a potential biomarker for inflammation. Neuroimage. 2008;40:43-52.

13. Price JC, Klunk WE, Lopresti BJ, et al. Kinetic modeling of amyloid binding in humans using PET imaging and Pittsburgh Compound-B. J Cereb Blood Flow Metab. 2005;25:1528-1547.

14. Benjamini Y, Hochberg Y. Controlling the false discovery rate: a practical and powerful approach to multiple testing. J R Stat Soc, B. 1995;57:289-300.

15. Owen DR, Yeo AJ, Gunn RN, et al. An 18-kDa translocator protein (TSPO) polymorphism explains differences in binding affinity of the PET radioligand PBR28. J Cereb Blood Flow Metab. 2012;32:1-5.

16. Mizrahi R, Rusjan PM, Kennedy J, et al. Translocator protein ( $18 \mathrm{kDa})$ polymorphism (rs6971) explains in-vivo brain binding affinity of the PET radioligand [ ${ }^{18}$ F]-FEPPA. J Cereb Blood Flow Metab. 2012;32:968-972.

17. Guo Q, Owen DR, Rabiner EA, Turkheimer FE, Gunn RN. Identifying improved TSPO PET imaging probes through biomathematics: the impact of multiple TSPO binding sites in vivo. Neuroimage. 2012;60:902-910.
18. Owen DR, Gunn RN, Rabiner EA, et al. Mixed-affinity binding in humans with 18-kDa translocator protein ligands. J Nucl Med. 2011;52:24-32.

19. Gavish M, Bachman I, Shoukrun R, et al. Enigma of the peripheral benzodiazepine receptor. Pharmacol Rev. 1999;51:629-650.

20. Coughlin JM, Wang Y, Ma S, et al. Regional brain distribution of translocator protein using $\left[{ }^{11} \mathrm{C}\right] \mathrm{DPA}-713$ PET in individuals infected with HIV. J Neurovirol. 2014;20:219-232.

21. Jučaite A, Cselenyi Z, Arvidsson A, et al. Kinetic analysis and test-retest variability of the radioligand $\left[{ }^{11} \mathrm{C}\right](\mathrm{R})-\mathrm{PK} 11195$ binding to TSPO in the human brain: a PET study in control subjects. EJNMMI Res. 2012;2:15.

22. Kropholler MA, Boellaard R, van Berckel BN, et al. Evaluation of reference regions for (R)-[ $\left[{ }^{11} \mathrm{C}\right] \mathrm{PK} 11195$ studies in Alzheimer's disease and mild cognitive impairment. J Cereb Blood Flow Metab. 2007;27:1965-1974.

23. Yasuno F, Ota M, Kosaka J, et al. Increased binding of peripheral benzodiazepine receptor in Alzheimer's disease measured by positron emission tomography with $\left[{ }^{11}\right.$ C]DAA1106. Biol Psychiatry. 2008;64:835-841.

24. Okello A, Edison P, Archer HA, et al. Microglial activation and amyloid deposition in mild cognitive impairment: a PET study. Neurology. 2009;72:56-62.

25. in 't Veld BA, Ruitenberg A, Hofman A, et al. Nonsteroidal antiinflammatory drugs and the risk of Alzheimer's disease. N Engl J Med. 2001;345:1515-1521. 